Juho Roponen, M.Sc., Systems Analysis Laboratory, Dept. of Mathematics and Systems Analysis, Aalto University School of Science; juho.roponen@gmail.com Ahti Salo, Professor, Systems Analysis Laboratory, Dept. of Mathematics and Systems Analysis, Aalto University School of Science, ahti.salo@aalto.fi

\title{
ADVERSARIAL RISK ANALYSIS FOR ENHANCING COMBAT SIMULATION MODELS
}

\begin{abstract}
Adversarial Risk Analysis (ARA) builds on statistical risk analysis and game theory to analyze decision situations involving two or more intelligent opponents who make decisions under uncertainty. During the past few years, the ARA approachwhich is based on the explicit modelling of the decision making processes of a rational opponent-has been applied extensively in areas such as counterterrorism and corporate competition. In the context of military combat modelling, however, ARA has not been used systematically, even if there have been attempts to predict the opponent's decisions based on wargaming, application of game theoretic equilibria, and the use of expert judgements. Against this backdrop, we argue that combining ARA with military combat modelling holds promise for enhancing the capabilities of combat modelling tools. We identify ways of combining ARA with combat modelling and give an illustrative example of how ARA can provide insights into a problem where the defender needs to estimate the utility gained from hiding its troop movements from the attacker. Even if the ARA approach can be challenging to apply, it can be instructive in that relevant assumptions about the resources, expectations and goals that guide the adversary's decisions must be explicated.
\end{abstract}

\section{Key words}

Adversarial Risk Analysis (ARA), Combat Modeling, Simulation

\section{Introduction}

Adversarial risk analysis (ARA) combines statistical risk analysis and game theory to provide appropriate methods for analyzing decision making situations which involve two or more intelligent actors who make decisions with uncertain outcomes. Such situations are encountered, for example, in counter-terrorism and corporate competition (Rios Insua et al., 2009). 
Traditional statistical risk analysis was developed to assess and mitigate risks in contexts where the loss is governed by chance (or Nature), for instance in the management of complex technological systems like nuclear power plants and the design of insurance policies against natural disasters. Apart from risks caused by such chance events, ARA seeks to capture risks caused by the self-interested and possibly malicious actions of intelligent actors: thus, modelling the decisionmaking behavior of these actors is central to ARA. These kinds of decision models can be based, for example, on classical game theory (Myerson, 1991) or psychological considerations (Camerer, 2003).

Yet game theory is not an ideal tool for describing and predicting human behavior. Minmax solutions-in which each actor seeks to minimize his expected losses across all the actions that are available to his opponents-can lead to unrealistic solutions, because real opponents do not usually follow the minmax rationality principle. Minmax solutions are also often difficult to compute in real situations, and they necessitate strong assumptions about what common knowledge the actors share (Kadane \& Larkey, 1982 and Meng et al., 2014). Moreover, the solutions can be overly pessimistic, because the mitigation of the worst possible scenario (which may have an extremely low probability) will induce the actors to make choices that a human opponent would not realistically make.

ARA has many obvious uses in military organizations. Much of the recent ARA literature has focused on counterterrorism, and many of the proposed ARA approaches can be applied to support military decision making. Zhuang and Bier (2007), for example, apply game theory to devise strategies for allocating resources between the protection from an intentional attack, on one hand, and from natural disasters, on the other hand. ARA methods can also be used to guide the allocation of resources between strategically important targets as well as the investment planning of military equipment and projects. Uses of ARA in finance and procurement are relevant, too, because military organizations acquire products and services from external contractors.

In this paper, we do not survey the broad ARA literature in view of military applications. Rather, we discuss how ARA can be applied to enhance combat modeling or to complement it. Specifically, we examine how ARA can be used to model the effects of military deceit, and how it can be used to aggregate results from different simulations to model a longer chain of events. 
The possibility of calculating the effects of military deception and its usefulness is one of the most promising ARA applications in combat modeling. Game theory has been applied to calculate the benefits of deceit before (Reese, 1980), but such applications are still rare. This is partly because the solutions of classical game theory presume that both sides have common knowledge about each other's goals and resources, which is not realistic when modeling deceit. ARA does not have this limitation. It can even be applied to calculate the usefulness of decoys and dummy systems, which makes it possible to estimate if these are worth the cost. Such estimation is very difficult if not impossible in most combat simulation models.

Using ARA to facilitate the simulation of longer chains of events holds promise, because simulation models are built to model combat on specific scale. For instance, simulation models which seek to accurately describe the combat between two tanks are ill-suited for modeling an entire battalion, and models for simulating fighting at the platoon level do not lend themselves well to the modeling an entire theater of operations. Still, with ARA it is possible combine results from several such simulation runs or even different simulation models to create a more encompassing optimization model. This can be very useful in stretching the limits of what can be done with existing simulation tools.

\section{Modeling adversarial risks}

In this section, we briefly describe how a situation in which there are adversaries whose actions affect each other's risks can be modeled. Our presentation builds largely on Rios Insua et al. (2009) who give a comprehensive presentation of ARA. For a good overview on how the ARA approach compares to classical game theory, we refer to Banks et al. (2011).

\section{Risk analysis}

The simplest form of a non-adversarial risk management problem is a situation in which the decision maker chooses one of the available decision alternatives whose costs are uncertain. This problem can be presented as an influence diagram as seen in Figure 1. 


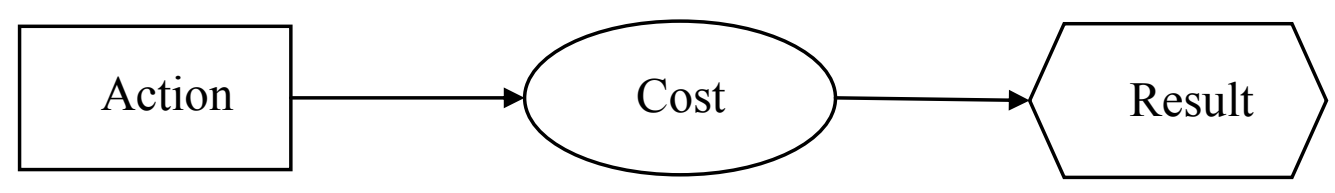

Figure 1: A simple influence diagram

An influence diagram is a directed acyclic graph with three kinds of nodes: rectangle shaped decision nodes, oval shaped uncertainty nodes, and hexagonal value nodes. Arrows pointing to value or uncertainty nodes indicate functional or probabilistic dependence, respectively. This means that the utility function at the value node depends on its immediately preceding nodes, and the probabilities associated with an uncertainty node depend are conditioned on the values of the immediately preceding nodes. Arrows which point to decision nodes indicate that the values of these preceding nodes are known at the time of the decision. (cf. Howard \& Matheson, 2005)

Figure 1 shows a situation where the decision maker has to make a decision $a$ from a set $A$ of possible choices, represented by the rectangle. The cost $c$ associated with this decision is uncertain and is modeled through the probability density function $\pi(c \mid a)$, represented by the oval node. The result is modeled by Von NeumannMorgenstern utility function $u(c)$. The decision maker seeks to maximize the expected utility

$\psi=\max _{a \in A}\left[\psi(a)=\int u(c) \pi(c \mid a) d c\right]$.

In practice, the costs of a particular action can be complex in that they can include both fixed and random terms. As a result, organizations seek to perform a risk assessment to better identify disruptive events, and to estimate their probabilities and associated costs.

\section{Adversarial risks}

We now consider a situation in which there are two adversaries (Attacker and Defender) whose decisions affect the risks that each faces. Figure 2 extends the influence diagram to include the adversary in a symmetrical situation in which the decisions of both parties affect the risks and costs that the other faces, and both 
seek to maximize their own expected utility. In this example, the roles are symmetric; but it is possible to model asymmetric scenarios as well by building asymmetric influence diagrams.

We denote the sets of possible actions of Attacker and Defender with $A$ and $D$ respectively. Their utility functions are $u_{a}(\cdot)$ and $u_{d}(\cdot)$. The sets containing their beliefs about different probabilities are $P_{a}$ and $P_{d}$. As can be seen in the influence diagram in Figure 2, one of the nodes, Hazard, is common to both sides. It can represent uncertainties which affect parties, such as weather for example. The other cost nodes-which are not common-represent random costs for both parties which can be very different for the parties.

The expected utilities for both the Attacker and the Defender depend upon the actions of both. Specifically, by extending on (1), we obtain the Attacker's expected utility for choosing action $a \in A$ when the Defender chooses action $d \in D$

$\psi_{A}(a, d)=\int u_{A}(c) \pi_{A}(c \mid a, d) d c$,

where $\pi_{A}(c \mid a, d) \epsilon P_{A}$ represents the Attacker's beliefs about his costs for the decision pair $(a, d)$. It is noteworthy that these beliefs do not necessarily have to match reality, because we are only modeling the Attacker's decision. The expected utility for the Defender is analogous.

This representation of ARA matches normal form games in which both players take simultaneous decisions. One could also build an influence diagram that represents sequential games, such as Stackelberg games, in which the players make their moves alternately. The ARA methodology can be applied to solve such games, too ( $c f$. Banks et al., 2011 and Rios \& Rios Insua, 2012).

\section{Bayesian framework for ARA}

A problem like the one presented in Figure 2 can be solved using classical game theory if the costs and utility functions of both players are common knowledge. However, if the players do not have correct and accurate information about the costs, resources, and goals of the adversary (which is often the case in reality), the Nash equilibrium solution does not exist. 


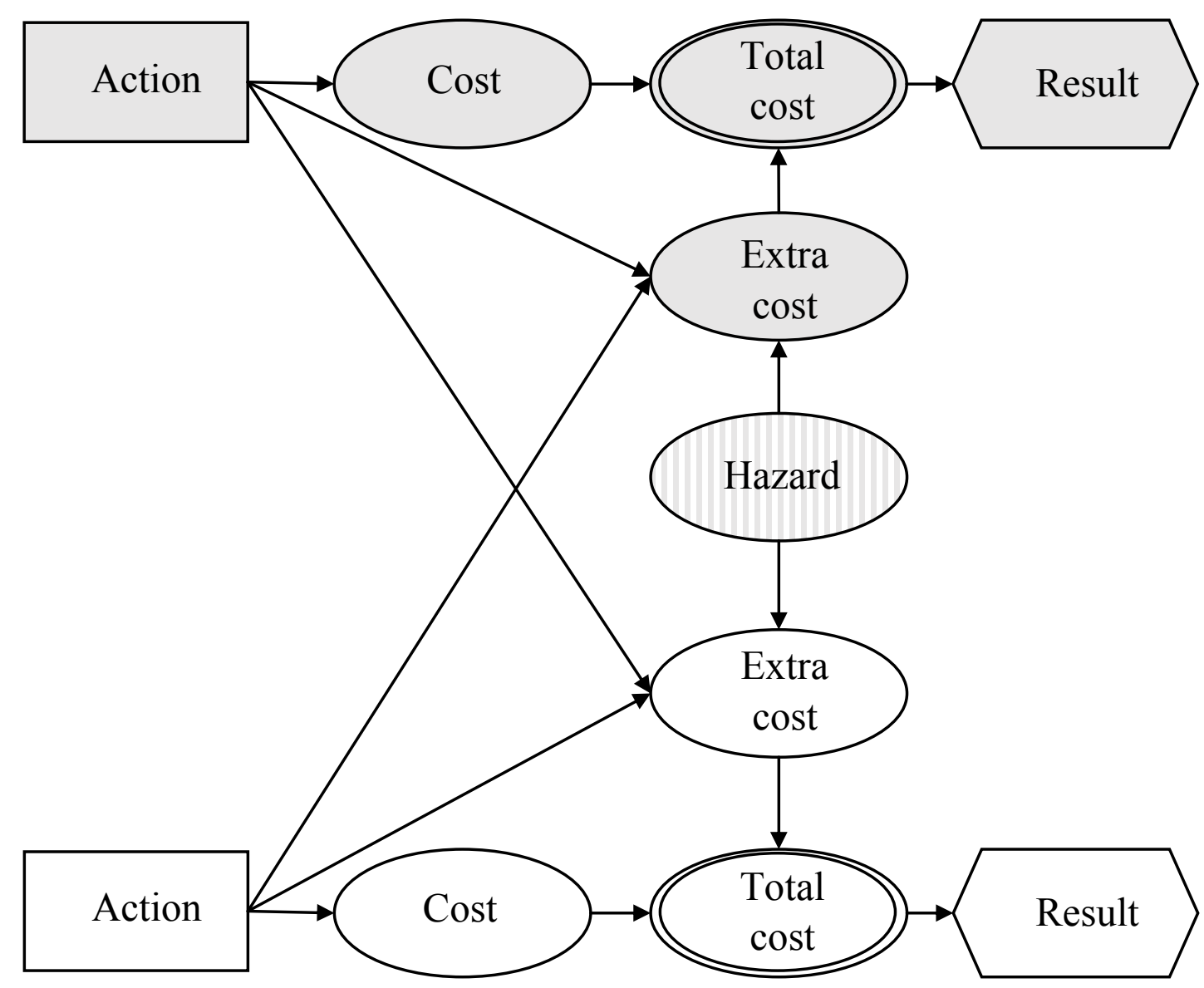

Figure 2: Influence diagram with an adversary

ARA solves this problem by using a Bayesian strategy to express uncertainty about the adversary's decision. From the Attacker's point of view, this means that the Defender's decision is a random variable as presented in Figure 3. To solve this problem, the Attacker needs more than just $\pi_{A}(c \mid a, d) \epsilon P_{A}$ and $u_{A}(c)$. Specifically, he also needs $p_{A}(d)$, which is the probability that the Defender chooses defense $d$ as estimated by the Attacker. To find that, the Attacker is assumed to use mirroring to form an estimate of the Defender's utility function $u_{D}(c)$ and the Defender's costs $\pi_{D}(c \mid a, d)$. In other words, the Attacker assumes that the Defender acts rationally and that the Defender uses a similar approach to predict the actions of the Attacker. 
If the Attacker tried to estimate the Defender's utility function and cost function by assuming that the Defender is doing exactly the same thing as what he is doing, the Attacker would need to think what the Defender thinks he thinks. To avoid infinite regress, the chain is usually cut here and the Attacker just forms an educated guess about the Defender's beliefs about the Attacker's estimated utilities and costs. In principle, this analysis could be taken even further, but usually this is not realistic.

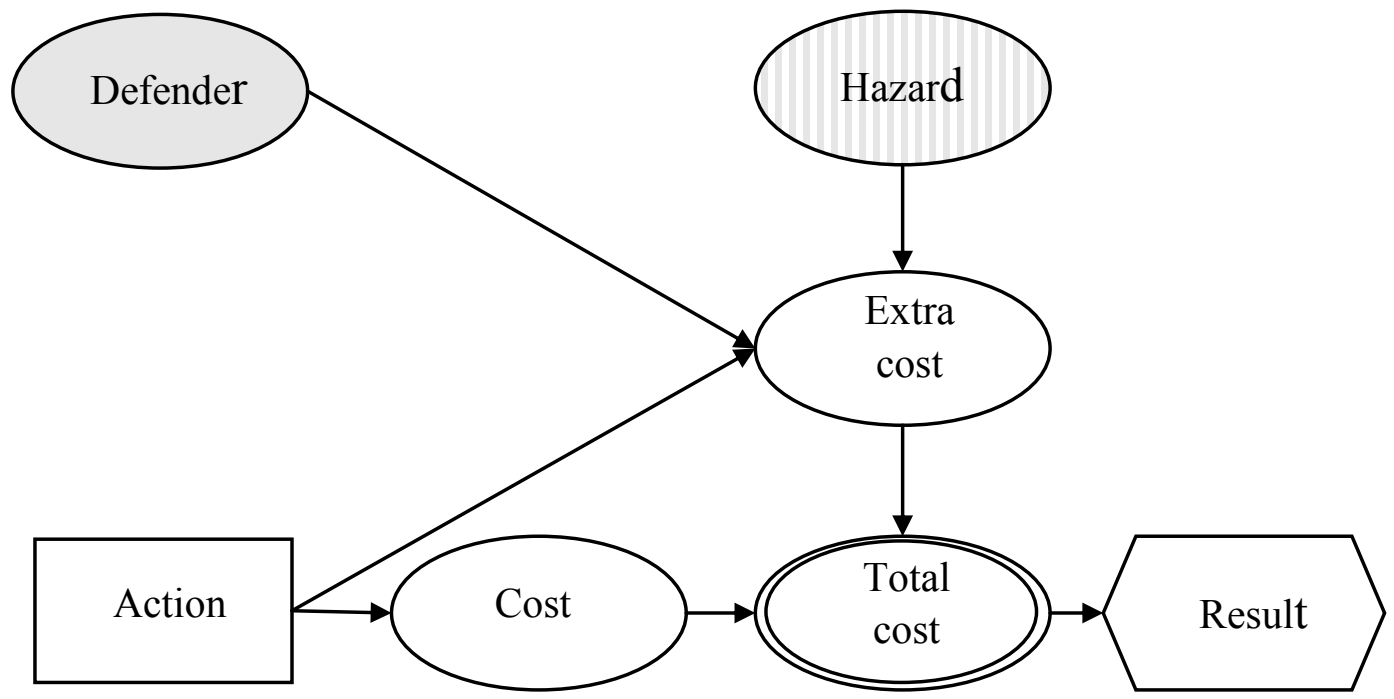

Figure 3: Influence diagram from the Attacker's point of view.

\section{Alternative approaches for modeling adversary's decision making}

The ARA methodology has analogues with Bayesian level-k thinking: specifically, our approach to modeling of the opponent's beliefs resembles level-2 thinking. Rothschild et al. (2012) have taken the approach further and applied level-k thinking to the ARA approach. Their methodology has drawbacks, though, because the level-k approach requires additional assumptions and the problems become quickly intractable due to their growing complexity. The greatest advantage of level-k thinking is that it shows how the level of adversary's thinking affects the optimal decision.

Caswell et al. (2011) present a model in which the decision process is evaluated using a Bayesian network with an embedded semi-Markov decision process. Compared to the ARA approach, their model can be used to present the adversary's 
decision process with greater accuracy. However, as in any decision analysis model, the results are only as good as inputs, and a detailed description of the adversary's thought process would require detailed information about the adversary's resources, values and goals.

Zuckerman et al. (2012) represent adversarial activity with a Beliefs-DesiresIntentions (BDI) based model; such models are commonly used to describe teamwork and cooperation. In this approach, the adversary can be modelled as a more nuanced rational agent instead of an omniscient utility maximizer. Yet, the model is not very elaborate, and it can be applied only in zero-sum games in which the goals are easily decomposable.

\section{Applying ARA to military combat modeling}

A significant proportion of ARA literature is focused on preventing terrorist threats and, more specifically, on how limited resources should be allocated to combat such threats ( $c f$. Pat-Cornell \& Guikema 2002, Kardes \& Hall 2005, Zhuang \& Bier 2007, Golany et al. 2009, and Kroshl et al. 2015). Nevertheless, in this section we focus more on how ARA can be applied to to military combat modeling and modeling processes, because resource allocation is well covered in earlier research.

We have chosen to examine what possibilities ARA offers for simulating longer chains of events and military deceit, because these are some of the more difficult problems to be handled with existing simulation and analysis tools. To some extent, these topics are interconnected, because deception and misinformation can have major impacts on what happens in the battlefield. Many of the following ideas are still untested, and they are presented as suggestions for worthy topics for future research.

\section{Simulating larger chains of events}

The ARA methodology can also be applied to model military operations that are too large to simulate as a single scenario. The scale can become an issue if the number of units involved is too large, or the operation takes place over such a long timeframe that the number of possible paths based on the events becomes excessive. Kangas and Lappi (2006) present how methods of probabilistic risk analysis can be used in conjunction with stochastic combat modeling to analyze longer chains of events. The ARA approach can be used to build on such results to take the analysis one step further. In addition to predicting the success chances of 
larger operations, it would be possible to predict which ones out of adversary's alternative actions can affect the chain of events most.

Furthermore, ARA can be used to expand a small scale simulation model to a larger scale optimization model. In practice this could mean, for example, using a platform level simulator that can model an aerial battle between fighter aircraft in conjunction with ARA to forecast which decisions would most likely lead to air superiority in the conflict. This approach is not even restricted to using a single simulation model. It would not be significantly more difficult to combine the simulation results from several different models.

Practically any combat model can be used with ARA methodology on condition that the probabilities for each side winning the battle as well as the expected losses on both sides can be calculated. This includes essentially all stochastic combat models and even some deterministic ones. The selection of the combat model must fit the problem at hand. Sometimes the best choice is a platform level Monte Carlo simulation, and sometimes it can be a high level attrition model like the FATHM (Fast Theater Model) (Brown \& Washburn, 2000).

In some cases, it is possible to use ARA to model longer chains of events without having to rely on an actual stochastic combat modeling software like Sandis (cf. Kangas and Lappi, 2006). There are also alternative, lighter stochastic computational models that can be used to predict the outcome of a duel between two platoon sized forces (Lappi et al., 2012; Åkesson, 2012; Roponen, 2013). These models can be used to significantly cut down the time for calculating all the success probabilities and the expected losses in different stages of the chain. There are also additional time savings from not having to create a complete model scenario, which, as noted earlier, is a time consuming process. The use of the lighter duel simulation methods could be automatized to a certain degree, because they require far fewer input parameters.

To offer a rough outline for how ARA can be applied to a longer chain of events, we present an example case in which modeling the events as a single combat simulation would likely be very time consuming and would not offer any real benefits over the ARA approach. That is, we examine a situation in which there are two bridges that can be used to cross a river. One party wants to cross the river and the other wants to prevent that from happening by using military force. The Defender has three platoons of soldiers available for defending the bridges. The Attacker has six platoons which try to get across. 
In order to examine a problem such as this, one first needs to a narrative of what chains of events are possible as a result of the decisions that the actors can take. To keep the problem tractable, the number of possible decision options as well as the end results of random events needs to be kept to a minimum, because the number of calculations required grows exponentially with each step. The problem has been presented as an influence diagram in Figure 4.

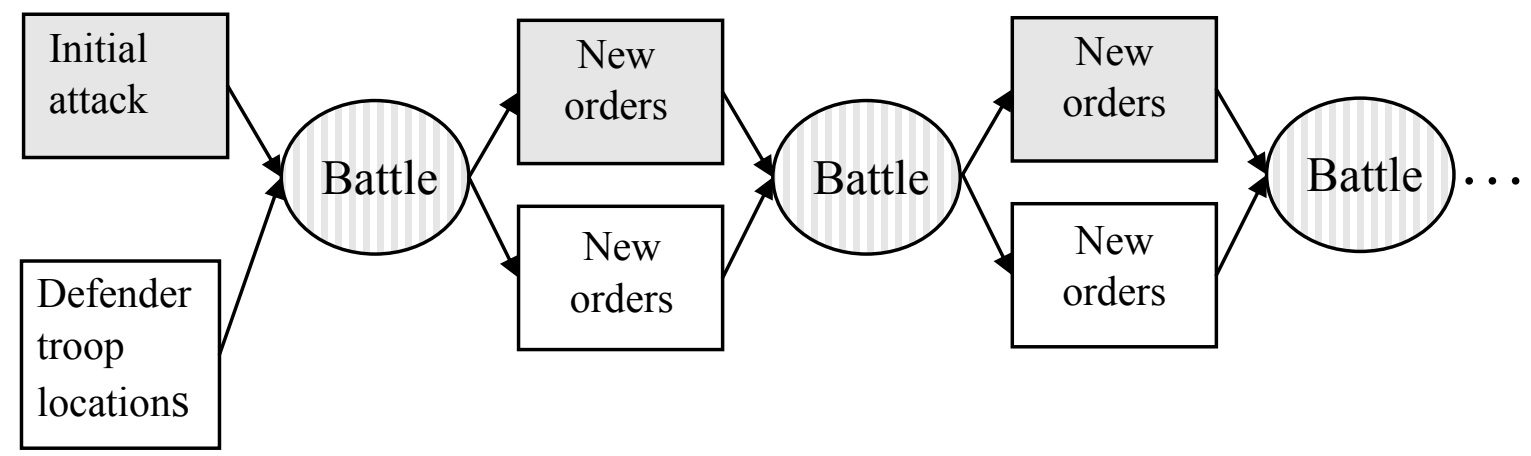

Figure 4: Influence diagram depicting a longer chain of events. The darker nodes are associated with the Attacker, as perceived by the Defender, as uncertainty nodes and vice versa. The striped nodes are uncertain events common to both sides.

The Defender's first decision is how he should deploy his troops initially. This includes deciding how he should divide his troops between the two bridges and reserves, but depending on the level of sophistication of the combat model used for the calculations other variables like the use of terrain, fortifications and mines could be included as well. The Attacker also needs to decide how he is going to use his troops for the initial attack. This includes the number of troops used and the target (or many targets) of the attack. Depending on the modeled situation the Attacker might be operating with very limited information. The initial attack might be used to just scout the Defender's strength.

The influence diagram in Figure 4 shows the two decisions as independent, but it does not really need to be so. Depending on which situation we wish to model, either the Attacker or the Defender could act after finding out what the other is planning to do. It is even possible to account for asymmetric information on both sides, arising for instance from greater familiarity with the terrain, military deception or some other reason. For the sake modelling parsimony, the number of 
possible choices should be kept as low as possible. This means, for example, that the troops should be deployed as whole platoons instead of single soldiers.

Once the possible initial decisions have been determined, the probabilities of all the possible results of the first battle for all the possible initial conditions need to be computed. The number of possible battle results can become computationally overwhelming, because all possible combinations of casualties on both sides are technically different outcomes. It is not feasible to calculate the next step if the number of possibilities is in the hundreds. The amount can be reduced to a more manageable number by assuming that the losses suffered by each side are conditional only on the result of the battle and not on each other. In this way, the possible results can be limited to wins and losses or some other smaller set. One way to choose the set of possible results is to take a look at the next step and identify which results would lead to different decisions and use those. Depending on the modeled chain of events, this can be easy, nearly impossible, or anything in between these extremes.

When the possible results of the first battle have been determined, we proceed to the decisions that follow it, then to the next battle, and so forth. In some these outcomes, either the Attacker or the Defender will not be able to continue effectively, which shortens the chain of events. For the sake of tractability, one also has to decide where these chains of events will have terminated so that there is no more fighting. It is possible that in more complex cases, the influence diagram is not ideal for visualizing the problem. Alternatively, the problem can be shown as a decision tree, because the ARA methodology is not tied to influence diagrams.

After all the possible chains of events have been elaborated and the corresponding possible end results have been determined, we proceed by calculating backwards from the end to estimate the probabilities of these results. Towards this end, we first solve the ARA problem formed by the last decisions in the chain and the ensuing battle. To find out the probability that the initial conditions for those ARA problems are met, we then solve the ARA problems formed by the battle and decisions preceding them and so forth until the first decisions have been analyzed. The utility function for each problem in the chain is formed from the maximized expected utility gained from each outcome as given by solving the ARA problems following it. 


\section{Modeling the effectiveness of military deceit}

"All warfare is based on deception." (Sun Tzu) Using deceit to gain an upper hand against an adversary is an absolutely integral part of military tactics and strategy. Still, the effects of deceit are very difficult to predict and simulate with existing operational analysis and combat modeling software. Because the effects cannot be readily reduced to mathematical formulas, modeling the effects of deceit relies usually on expert judgements. In the context of combat modeling, this usually means that the required expert opinions are provided by the operator of the simulation tool.

A common alternative is to use wargames to model the uncertainties in human decision making; but even this approach also has problems as wargames ignore many aspects of reality. Questions of solvability do not arise in wargames, because the aim is not to determine optimal tactics. Rather, realizing that wars are fought by humans, wargames study the decision process of humans. One problem in this approach is that in a game, the player can make decisions that he would not really make as long as these decisions produce good results in the simulation. For example, in a simulated environment casualties may not have the weight that they would have in actual combat. The second problem is that wargames often capture typical decision making behavior (rather than optimal decisions) because the players play a small number of games only. Thus, for instance, the resulting lack of repetition may overstate the effectiveness of new weapon systems, because the opponent does not have time to learn and adapt his tactics to counter these systems. To some extent, the lack of repetition may be deliberate due to the fear that the players would learn to use the artificialities of the wargame to their advantage instead of developing better military strategies. Another reason for the lack of repetitions is that wargaming is time consuming and expensive. (Washburn \& Kress, 2009, 111-130)

The ARA approach could be used to assess the effects of deceit tactics on the decision making of the adversary. Specifically, the ability to model the effects of the adversary's altered perceptions would be a useful complement to the elicitation of expert judgements. Mathematical equations are, after all, immune to effects of optimistic thinking.

ARA can be used relatively easily to model situations in which the adversary is deliberately misinformed about the strength or capabilities of the opponent, for instance as a result of hiding troop movements and employing dummy units or 
decoys. Then, ARA helps estimate the effect the misinformation on the adversary's decision making and whether this effect is beneficial so that the benefits outweigh the costs. In the next section, we give an example of such an estimation process.

The ARA approach is not limited to deceit that happens on the battlefield; indeed, military deceit is pervasive in military planning and decision making. In theory, ARA can be used to study the effects of almost any misinformation, but usually these effects can not be modeled with combat simulation models (except in the case of misinformation that relates to the number or capabilities of weapon systems, sensor systems or military units). While the ARA methodology does not give tools for predicting the probability with which deception will succeeds, it helps assess the possible effects of successful deception may be, which helps decide what information is worth hiding or altering.

\section{Example of applying ARA to a military deceit problem}

To demonstrate how ARA can be applied, we use it here to analyze a relatively simple tactical problem which illustrates some of the key aspects of the approach.

\section{The problem}

Consider the following situation in which there are two adversaries: the Defender and the Attacker. The Defender has two valuable targets he needs to protect, Target 1 and Target 2. The Defender has a total of 60 troops, 40 of whom are situated at Target 1 and 20 at Target 2 . The Defender can try to secretly move troops from one target to another, but there is a possibility that the Attacker will notice the troop movement. After the Defender has moved the troops he wants to move, the Attacker will decide which target he will attack. If the Defender succeeds in moving the troops without the Attacker noticing, the Attacker will have to decide his target using incomplete information. We solve this problem from the point of view of the Defender. Figure 5 shows the influence diagram from the Defender's point of view. 


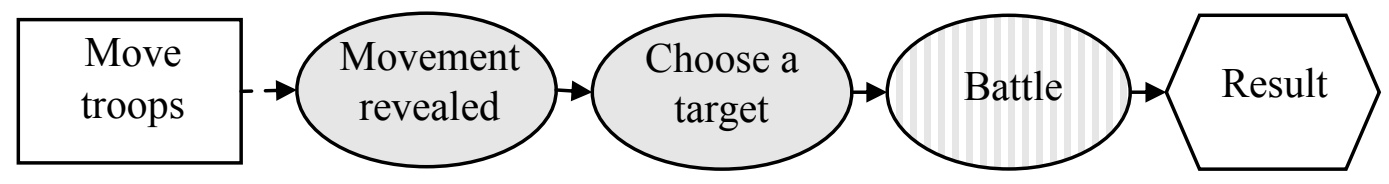

Figure 5: Influence diagram of the example case from the point of view of the Defender.

We denote the set of options the Defender as $D$, which represents number of squads of five soldiers moved from Target one to Target 2, and the options of the Attacker $A=\{1,2\}$ which represents the choice between the two targets. The uncertainties in this case are whether the troop movement is revealed to the attacker $R(R=1$ if it is and $R=0$ if not) and the outcome of the battle $S(S=1$ if the Defender wins and $S=0$ if the Defender loses). The utility functions over the costs are $u_{D}\left(c_{D}, c_{A}\right)$ and $u_{A}\left(c_{D}, c_{A}\right)$, with costs dependent on the actions of the Defender and the Attacker.

The Defender wants to solve

$$
\psi=\max _{d \in D}\left[\psi(d)=\sum_{a \in A} \sum_{r \in R}\left[p_{D}(r) p_{D}(a \mid d, r) \psi_{D}(a, d)\right] .\right.
$$

In order to solve this problem, it is necessary to assess the probabilities over the costs, conditional on $(a, d, S)$; and about $S$, conditional on $(a, d)$. In this case, the Attacker and the Defender have different assessments: for example, for success, these are $p_{D}(S=1 \mid a, d)$ and $p_{A}(S=1 \mid a, d)$, respectively. It is likely that the Attacker's assessment of the success of the assault differs from that of the Defender, because the Attacker may not know the Defender's decisions (see Figure 6). The expected utility for the Attacker, resulting from $(a, d)$ is

$$
\begin{aligned}
\psi_{A}(a, d) & =p_{A}(S=0 \mid a, d) \sum_{c_{A}} \sum_{c_{D}}\left[u_{A}\left(c_{A}, c_{D}, a, S=0\right) \pi_{A}\left(c_{A}, c_{D} \mid a, d, S=0\right)\right] \\
& +p_{A}(S=1 \mid a, d) \sum_{c_{A}} \sum_{c_{D}}\left[u_{A}\left(c_{A}, c_{D}, a, S=1\right) \pi_{A}\left(c_{A}, c_{D} \mid a, d, S=1\right)\right] .
\end{aligned}
$$

The Defender's expected utility is similar. 


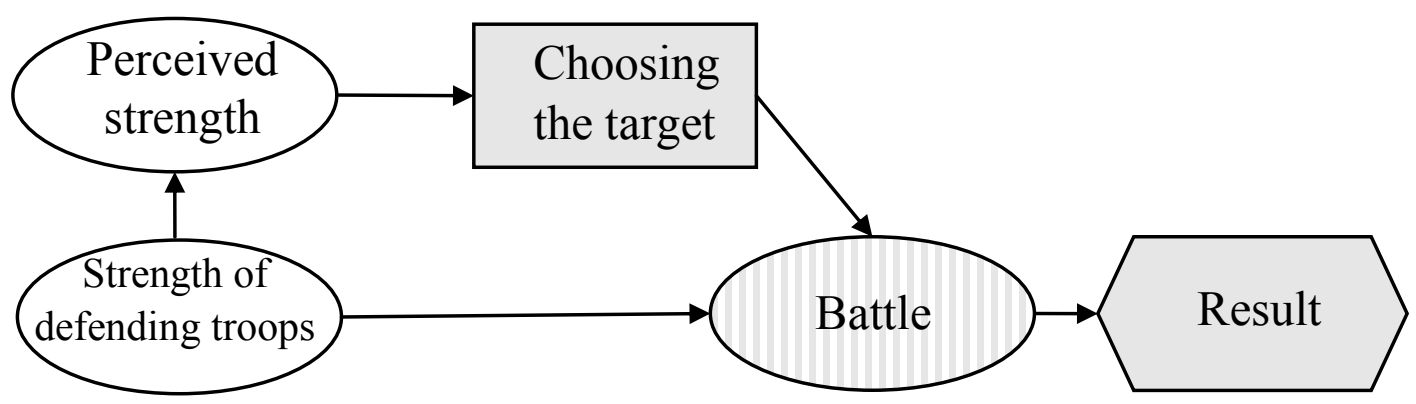

Figure 6: Influence diagram of the example case from the point of view of the Attacker.

We now solve the game from the Defender's point of view. The Defender has 40 men defending Target 1 and 20 men defending Target 2. The Defender considers value of Target 1 to be 50 and value of Target 2 to be 30 . He considers the value of a single combat ready soldier to be 1 . He has the option moving troops between the targets without the Attacker's knowledge. There is, however, a probability $p_{R}$ that the attacker finds out about the troop movement. The Defender also estimates that the Attacker has at least 20 men but no more than 35, and he thinks that the most likely number is 30 , so he fits a triangular distribution.

Using the strength estimates of both forces, the Defender can use, for example, a stochastic combat model to calculate $p_{D}$ and $\pi_{D}$. Specifically, the Defender assesses that the utility in this situation follows the function

$$
u_{D}\left(S, a, c_{A}, c_{D}\right)=\left\{\begin{array}{cc}
0.1 c_{A}-c_{D}, & \text { if } S=1 \\
-50+0.1 c_{A}-c_{D}, & \text { if } S=0 \text { and } a=1 \\
-30+0.1 c_{A}-c_{D} & \text { if } S=0 \text { and } a=2
\end{array}\right.
$$

where $S=1$ corresponds to the situation where the Defender manages to hold the target area, $a=1$ corresponds to the situation where the Attacker decides to attack Target 1 and $a=2$ corresponds to the Attacker deciding on Target 2, and $c_{A}$ and $c_{D}$ are the Attacker's and the Defender's losses respectively.

Solving the problem for the Defender is not sufficient for determining $p_{D}, \pi_{D}$ and $u_{D}$. To calculate the expected utility from decision $d$, he first needs to estimate $p_{D}(a \mid d, r)$. Towards this end, the Defender needs to solve the problem from the viewpoint of the Attacker. He assumes that the Attacker, too, is an expected utility maximizer. The problem is presented from the Attacker's point of view in Figure 6. 
The Defender estimates that the Attacker thinks the Defender has 36 to 44 men at Target 1 and 18 to 22 men at Target 2 (with all values equally probable), and the Attacker has probability $p_{D}(R=1)=0.1$ of finding out about the Defender's troop movement. If the Attacker detects the Defender moving troops, he will be able to accurately count the number of troops moved. Using those strengths for his estimates he can use the same stochastic combat model used to solve $p_{D}$ and $\pi_{D}$ to calculate $p_{A}$ and $\pi_{A}$.

The Defender estimates that the Attacker's utility function is similar to his own. However, the Defender does not know for sure how valuable each target is to the Attacker. He models this uncertainty by adjusting the weights of successful capture of each target $S$. Thus, he estimates that the Attacker's utility function is

$$
u_{A}\left(S, a, c_{A}, c_{D}\right)=\left\{\begin{array}{cc}
0.1 c_{D}-c_{D}, & \text { if } S=1 \\
50+U_{1}+0.1 c_{D}-c_{A}, & \text { if } S=0 \text { and } a=1 \\
30+U_{2}+0.1 c_{D}-c_{A} & \text { if } S=0 \text { and } a=2,
\end{array}\right.
$$

where the distributions $U_{1}$ and $U_{2}$ are uniformly distributed over the interval $[-5,5]$.

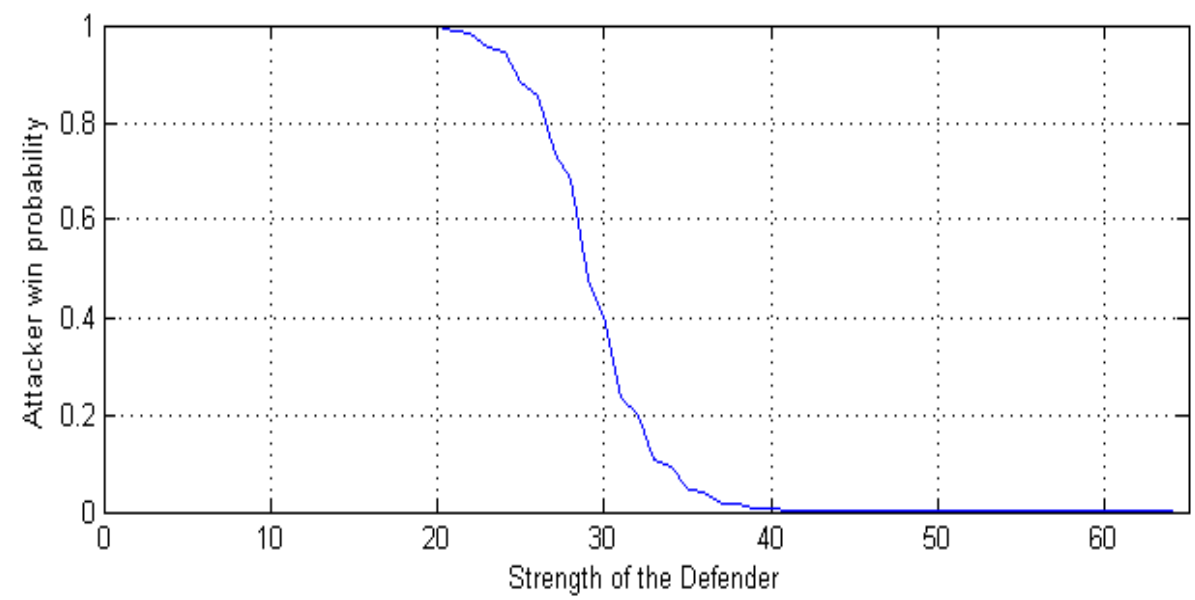

Figure 7: The Attacker's win probability with strength of 30 as a function of the Defender's strength. The minor perturbations in the curve are caused by the assumption that the battle is lost when the unit has lost half of its troops, which means that even strengths are slightly less advantageous for the Defender.

We solve the problem step by step. First, the Defender will: 
1. Calculate the success probabilities and expected losses for both sides for all the possible combinations of strengths of both sides (Figure 7).

2. Calculate the Attacker's expected utilities $\psi_{A}$ for attacking each target for all possible strengths of the Attacker's force taking into account the uncertainties with $u_{A}$ the fact that the Attacker has knowledge of the Defender's troop movement on probability $p_{D}(R=1)$.

3. Compare the expected utilities to get an estimate for the probability of an attack on each target for each possible strength of the Attacker.

4. Consider the probability of an attack with a specific strength of the attacker and the probability for each of those strengths to calculate $p_{D}(a \mid d, r)$.

5. Calculate $\psi_{D}(a, d)$.

6. Use $p_{D}(a \mid d, R)$ and $\psi_{D}(a, d)$ to determine the decision $d$ which maximizes his expected utility.

We used the approximative method in Roponen (2013) to simulate a duel between two forces in order to calculate the probabilities in $\left(p_{D}, \pi_{D}, p_{A}\right.$ and $\left.\pi_{A}\right)$ in step 1, because the details for this method are publicly available and it produces the results of battles between two infantry units very efficiently. This program code was used to examine the win probabilities for all possible strengths of both sides. Situations in which a tie was predicted were counted as the Defender's victory, because the Attacker would be unable to capture the target. Moreover, a unit was assumed to lose the battle if it lost half of its fighting strength, which is the cause behind the roughness of curves depicted in Figures 7 and 8, because the unit strengths are nonnegative integers and odd numbers are not divisible by two.

We then wrote a program code to search the remaining steps exhaustively, to calculate the expected utilities, and to determine the attack probabilities $p_{D}(a \mid d)$. Because the Attacker perceived that target 2 was significantly weaker, he always chose to attack this target unless he found out about the troop movement, in which case he chose the target with actually higher expected utility. The attack probability on target 1 thus varied between 0 and 0.1 . Then the expected utilities of the Defender were calculated from $p_{D}$ and $\pi_{D}$ as seen in Figure 8. 


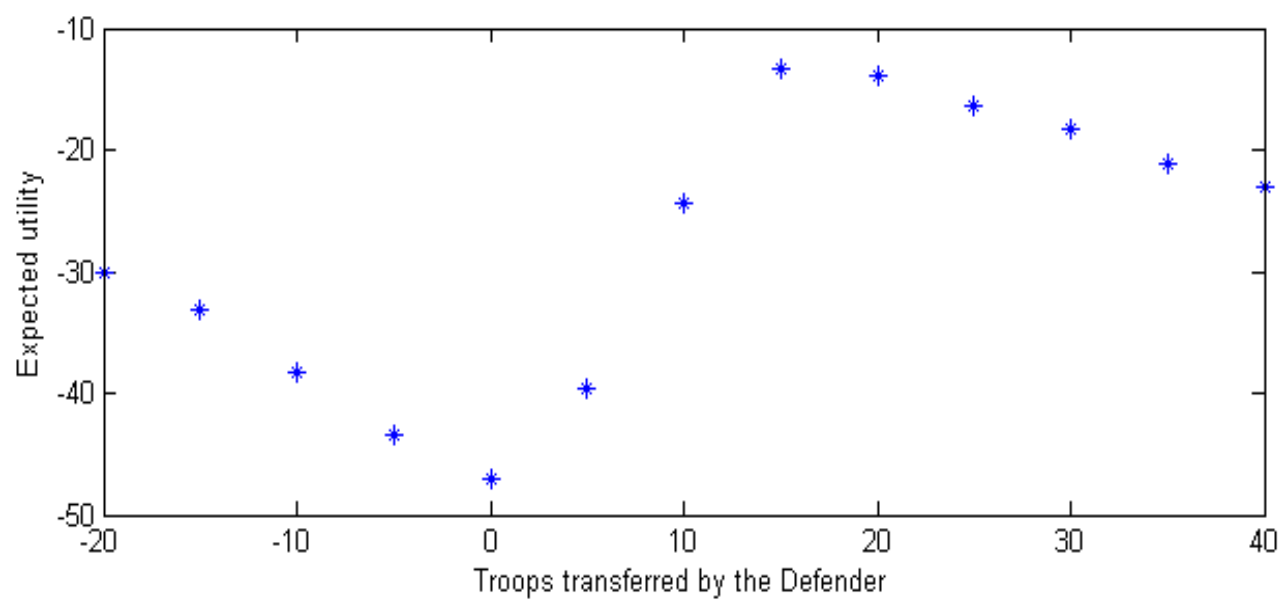

Figure 8: The expected utility gained by the Defender as a function of troops transferred from target 1 to target 2 .

The highest expected utility gained by the Defender, $\psi_{D} \approx-13.4$, was achieved by transferring three squads or 15 soldiers from target 1 to target 2 . This gave the Defender 0.933 probability of winning the battle if the Attacker chose to attack target 2 (which would happen if he does not notice the troop movement) and 0.281 probability of winning if the Attacker chose to attack target 1.

\section{Future research}

Adversarial risk analysis (ARA) is a relatively new research area which is becoming more prominent in the context of counter-terrorism and corporate competition. In this paper, we have discussed and illustrated the application of ARA to the modelling of longer chains of events and the effects of military deceit. There are also many other possible ways of applying the ARA methodology to combat modeling.

Arguably the most important reason for military combat modeling is that it provides support for strategic, tactical or technical decision making (Tolk, 2012, 55-78). However, it is not straightforward to translate the results of combat models into decision recommendations (Davis \& Blumenthal, 1991). One accessible way of using ARA in the context of simulation is to perform an exhaustive portfolio analysis of all relevant strategies. Such methods have been used to assess the costefficiency of different combinations of weapons systems, for example (Kangaspunta et al., 2012). 
In the same vein, ARA could be used to predict the most likely responses of the adversary and to calculate the expected utilities of each strategy under different conditions. The applicability of this approach depends how well the process can be streamlined and automated so that informative results about strategies can be provided more quickly than through manual analyses of combat simulations.

To enrich the possibilities of using combat simulation models, ARA could also be used to reduce the need for user interaction within existing combat modeling tools. Simple adversarial intent models have been used in professional wargaming to simulate intelligent forces (Santos \& Zhao 2006). This notwithstanding, most combat models do not yet include algorithms that would represent the human thought processes involved in tactical or strategic decisions (Washburn \& Kress, 2009, 111-130). Depending on the model, practically all higher level decisions concerning the position and strength of forces are made by the operator. As a result, the time required to create a scenario is usually significantly longer than the time required to calculate the results (Lappi, 2012).

The development of simulation models in which the units are able to make simple tactically sensible decisions would widen the range of problems that can be analyzed by using approaches such as data farming. The ARA methodology could then be used as a basis for these kinds of algorithm. Here, ARA has advantages over using game theory, because it accounts for uncertainties and even misinformation.

However, ARA cannot be readily applied to very low level or continuous decision making, because the required calculations would become just too overwhelming. ARA can be used most effectively in situations where the attention can be restricted to choices among relatively few possible strategies. If need be, it may be possible to simplify the problem by restricting attention to some plausible chains of events instead of calculating all possible chains of events. Analogues approaches towards simplification have been employed in constructing artificial intelligence systems for games such as Go and Chess, and they have been applied even in video game AI development (Churchill et al. 2012).

\section{Conclusions}

The ARA methodology has already found many uses in analyzing counter terrorism and corporate competition (Rios Insua et al., 2009). In this paper, we have discussed the relevance of this methodology to military combat modeling and 
presented concrete examples of how it can be applied. Specifically, we have outlined possible uses for the ARA approach in the context of modeling deceit and using ARA to stretch the limits of existing combat models to model longer chains of events. Many of these ideas are tentative and call for more research before they can be implemented into existing simulation. Another challenge is that real battles are extremely complex, they involve thousands of decisions, and the goals and resources are highly uncertain. Still, by focusing on the most important decision situations and decision alternatives can provide valuable insights.

We also presented an illustrative example in which ARA was combined with stochastic combat modeling to calculate the effects of military deceit. In this example, most calculations for solving the ARA part of the model were relatively straightforward and could be implemented into software code (there are numerous tools for calculating the results of battles; see, Kangas, 2005). We therefore believe that it possible to develop software tools for considerably more complex problems in which the dependencies between the adversaries' utilities and their decision behaviour are explicitly modelled. More generally, there is much potential in using the ARA approach to tackle realistic problems through stochastic combat modelling. This would serve to push the boundaries of ARA modelling in an important application area.

Fundamentally, ARA has much to offer for military combat modeling, because it is able to combine the conventional statistical approach of risk analysis-which is already widely employed in combat modeling-with fresh game-theoretical perspectives that help predict what one's opponents are likely to do. ARA can also be used to build optimization models on top of existing simulation models, which gives possibilities for new uses for these simulation models as well as new ways of visualizing the results of such models.

\section{References}

Banks, D., Petralia, F., \& Wang, S. (2011). Adversarial risk analysis: Borel games. Applied Stochastic Models in Business and Industry 27(2), pp. 72-86.

Brown, G. \& Washburn, A.R. (2000 rev. 2004). The fast theater model (FATHM), Project Report, (NPS-OR-01-002-PR), Naval Postgraduate School, Monterey, CA. Camerer, C. (2003). Behavioral Game Ttheory: Experiments in Strategic Interaction. Princeton University Press, New Jersey.

Caswell, D. J., Howard, R. A., \& Paté-Cornell, M. E. (2011). Analysis of national strategies to counter a country's nuclear weapons program. Decision Analysis, 8(1), $30-45$. 
Churchill, D., Saffidine, A., \& Buro, M. (2012). Fast Heuristic Search for RTS Game Combat Scenarios. In AIIDE.

Davis, P. K., \& Blumenthal, D. (1991). The base of sand problem: A white paper on the state of military combat modeling (No. RAND/N-3148-OSD/DARPA). Defense Advanced Research Projects Agency, Arlington VA.

Golany, B., Kaplan, E. H., Marmur, A., \& Rothblum, U. G. (2009). Nature plays with dice-terrorists do not: Allocating resources to counter strategic versus probabilistic risks. European Journal of Operational Research, 192(1), 198-208.

Howard, R. A. \& Matheson, J. E. (2005). Influence diagrams. Decision Analysis, 2(3), pp. 127-143.

Kadane, J. B. \& Larkey, P. D. (1982). Subjective probability and the theory of games. Management Science, 28(2), pp. 113-120.

Kangas, L. (2005). Taistelun stokastinen mallinnus. Master's Thesis, Helsinki University of Technology. http://sal.aalto.fi/publications/pdf-files/tkan05.pdf Accessed: 201405-07

Kangas, L. \& Lappi, E. (2006) Probabilistic risk analysis in combat modeling. In:

Hämäläinen, J. (ed.) Lanchester and Beyond. A Workshop on Operational Analysis Methodology. PVTT Publications 11

Kangaspunta, J., Liesiö, J., \& Salo, A. (2012). Cost-efficiency analysis of weapon system portfolios. European Journal of Operational Research, 223(1), pp. 264275.

Kardes, E., \& Hall, R. (2005). Survey of literature on strategic decision making in the presence of adversaries. Unpublished report.

Kovenock, D., Roberson, B. (2010). Conflicts with multiple battlefields, CESifo working paper: Empirical and Theoretical Methods, No. 3165

Kroshl, W. M., Sarkani, S., \& Mazzuchi, T. A. (2015). Efficient allocation of resources for defense of spatially distributed networks using agent-based simulation. Risk Analysis (to appear).

Lappi, E. (2012). Computational methods for tactical simulations. Julkaisusarja 1. N:o 1/2012. Doctoral Thesis, National Defence University, Finland

Lappi, E., Pakkanen, M., \& Åkesson, B. (2012). An approximative method of simulating a duel. In: Proceedings of the Winter Simulation Conference, WSC '12, pp. 208:1208:10

Lappi, E., Pentti, J., Åkesson, B., Roponen, J., Valtonen, J., Koskinen, J., Burhan, U., Sivertun, Å., and Hämäläinen, J. (2015). Team 4: Data farm. manuscript.

Meng, S., Wiens, M., \& Schultmann, F. (2014). A game-theoretic approach to assess adversarial risks. In: Brebbia, C.A. (ed.), Risk Analysis IX, WIT Press, Southampton, UK, pp. 141-152.

Myerson, R. B. (1991). Game Theory: Analysis of Conflict. Harvard University Press, Cambridge, MA. 
Paté-Cornell, E., \& Guikema, S. (2002). Probabilistic modeling of terrorist threats: A systems analysis approach to setting priorities among countermeasures. Military Operations Research, 7(4), pp. 5-23.

Reese, W. (1980). Deception in a game theoretic framework. In: Daniel, D. C., Herbig, K. L., Reese, W., Heuer, R. J., \& Sarbin, T. R. (1980). Multidisciplinary Perspectives on Military Deception (No. NPS-56-80-012A). Naval Postgraduate School, Monterey, CA.

Rios Insua, D., Rios, J., \& Banks, D. (2009). Adversarial risk analysis. Journal of the American Statistical Association, 104(486), pp. 841-854.

Rios, J., \& Rios Insua, D. (2012). Adversarial risk analysis for counterterrorism modeling. Risk analysis, 32(5), pp. 894-915.

Roponen, J. (2013). Kaksintaistelun approksimatiivinen mallintaminen. Bachelor's Thesis, Aalto University. http://sal.aalto.fi/publications/pdf-files/trop13_public.pdf Accessed: 2014-05-07

Rothschild, C., McLay, L., \& Guikema, S. (2012). Adversarial risk analysis with incomplete information: A level-k approach. Risk Analysis, 32(7), pp. 1219-1231

Santos Jr, E., \& Zhao, Q. (2006). Adversarial models for opponent intent inferencing. In: Kott A., \& McEneaney W. (2006) Adversarial Reasoning: Computational Approaches to Reading the Opponents Mind. Chapman \& Hall/CRC, Boca Raton, $1-22$.

Tolk, A. (2012). Engineering Principles of Combat Modeling and Distributed Simulation. Wiley. USA.

Tzu, S. (2004 print) The art of War. Trans. Giles L. Project Gutenberg.

Washburn, A. R., \& Kress, M. (2009). Combat Modeling. Springer. Heidelberg.

Zhuang, J., \& Bier, V. M. (2007). Balancing terrorism and natural disasters-defensive strategy with endogenous attacker effort. Operations Research, 55(5), pp. 976-991.

Zuckerman, I., Kraus, S., \& Rosenschein, J. S. (2012). The adversarial activity model for bounded rational agents. Autonomous Agents and Multi-Agent Systems, 24(3), 374409.

Åkesson, A. (2012). Automatic calculation of win probabilities and conditional strength distribution of units in stochastic simulation model. Master's Thesis, Åbo Akademi University. 